\title{
Investigation of Nonlinear Phenomenon for Insertion Type Integrated Magnetics Used in Linear Level Control Converters
}

\author{
W.Water ${ }^{1}$ Member, IEEE, J. Lu ${ }^{1}$, Senior Member, IEEE and N. Wang ${ }^{2}$, Member, IEEE \\ ${ }^{1}$ Queensland Micro- and Nanotechnology Centre, Griffith University, Nathan, QLD 4111 AU \\ ${ }^{2}$ Microsystems Centre, Tyndall National Institute, University College Cork, Cork, IRL
}

\begin{abstract}
This paper examines the nonlinear behavior of inserted type integrated magnetics used in Linear Level Control (LLC) converters. Due to the nonlinear behavior of the magnetics, implementation of the insertion type magnetic integration needs to be carefully considered. The saturation of magnetics is normally not a concern in high voltage input applications, but becomes a serious issue when designing for the low voltage input and high power applications. In this paper, the inserted type integrated magnetics has been categorized into two types according to their magnetic integration configurations. Investigation and discussion of their nonlinear behavior will be presented with the assistance of 3-Dimensional (3-D) simulation results based on the Finite Element Method (FEM). A case study of a $350 \mathrm{~W}$ DC-DC converter is used throughout the paper for the ease of illustration.
\end{abstract}

Index Terms - LLC resonant converter, finite element methods, magnetic integration, planar transformer.

\section{INTRODUCTION}

$\mathrm{L}$ LC resonant converters have attracted great interest in past decades due to pressures from the power electronic industry. However, it is only in recent years that such converters have been more widely introduced onto the market due to difficulties with the controlling circuit design [1]. To achieve Zero Voltage Switching (ZVS) or Zero Current Switching (ZCS), a resonant tank is required in the front end of the converter [2]. The resonant tank is composed of the resonant inductor $L_{s}$, the magnetizing inductor $L_{p}$ and the resonant capacitor $C_{s}$ (refer to Fig. 2 (a)). The $L_{s}$ is normally obtained by utilizing the leakage inductance $L_{e q}$ of the transformer in LLC circuitry. However, a greater $L_{s}$ is needed in some circumstances such as to reduce the operating frequency, or to better suppress the impulse current or voltage input source. Thus, magnetic integration was introduced, which effectively increases the $L_{s}$ required while keeping the total size of the magnetics small [3]-[7]. Among these integration structures, the inserted type is the most compact and inexpensive configuration due to its inherent geometry characteristics [8].

However, a partial magnetic saturation phenomenon is found when the introduced magnetic structure is implemented in low voltage input and high power applications. The inserted type integration transformer is partially saturated with high flux density circulating in the inserted magnetics. To investigate this nonlinear phenomenon, the inserted magnetic integration has been categorized into two types of structures in this paper. They are the Magnetic Insertion in Parallel (MIP) structure (Fig. 1(a)) [6] and the Magnetic Insertion placed Orthogonally (MIO) structure (Fig. 1(b)) [8]. The discussion and investigation of this nonlinear phenomenon is based on the practical experiments, mathematical verification and computer assisted FEM 3D modeling.

Manuscript received March 16, 2015. Corresponding author: W. Water (e-mail: w.water@griffith.edu.au).

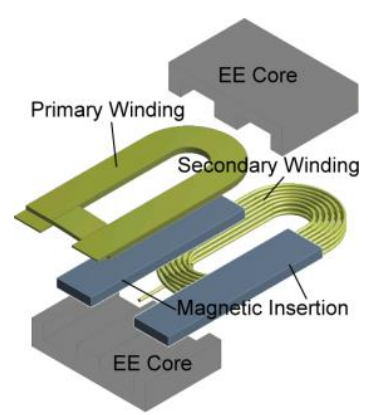

(a) MIP structure

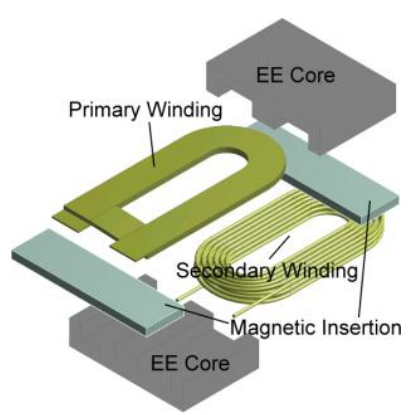

(b) MIO structure
Fig. 1. Explorer view of (a) MIP structure, and (b) the MIO structure

\section{CONSIDERATION OF THE NONLINEAR PHENOMENON}

\section{A. Equivalent Circuit Model for Integrated Magnetics}

In a conventional transformer, the $L_{e q}$ has a linear characteristic. There is no magnetic saturation concern because the air medium has no limit on the amount of flux it can handle. However, this phenomenon changes since a greater $L_{e q}$ becomes essential in the resonant topology to avoid high switching losses. In order to increase the $L_{e q}$, the magnetic material is normally utilized as the electro-magnetic force conduction medium, which generates the same magnetic force but requires less excitation energy. Due to the inherent nonlinear characteristics of the magnetic material, the LLC resonant circuit shown in Fig. 2 (a) can be simplified and adapted as shown in Fig. 2 (b), in which the $L_{s}$ and $L_{p}$ are represented with a nonlinear symbol.

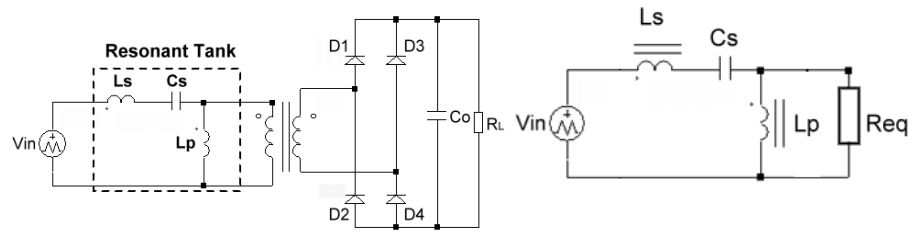

(a) Schematic

(b) New equivalent circuit

Fig. 2. LLC resonant circuit 


\section{B. Numerical EM Computation for Integrated Magnetics}

The nonlinear magnetic field of the magnetic integration can be described by the following equation,

$$
\nabla \times v \nabla \times \boldsymbol{A}-J=0
$$

where $\boldsymbol{A}$ is the magnetic vector potential, $v$ is the reluctivity and $J$ is the current density, which includes exciting current density and eddy current density. The energy function is generalised from linear techniques. Galerkin's method is used to discretize the governing equation. A system matrix equation can be obtained from ( 1 ) and rewritten as shown in ( 2 ), where the matrix $[\mathrm{S}]$ is the global coefficient matrix. $[\mathrm{M}]$ is the frequency harmonic matrix, $[\mathrm{K}]$ is the related current density and $\mathrm{G}$ is the weighted residual.

$$
G=[S]\{A\}+[M]\{A\}-\{K\}
$$

\section{CASE STUdy OF A $350 \mathrm{~W}$ LLC CONVERTER}

To examine the nonlinear phenomenon of magnetic integration, a case study example of a $350 \mathrm{~W}$ LLC converter is used, in which the parameters are tabulated in Table I. Two transformer prototypes were built with EE38/16/25 (L/W/H respectively in $\mathrm{mm}$ ) cores along with a magnetic insertion size of 8/2/36 (L/W/H). Both integrated prototype transformers were built according to the impedance requirements listed in Table I to ensure the experiment results are consistent and effective. Magnetic material of MnZn TP4A is chosen, and it is well suited for application frequency ranges between 100 $\mathrm{kHz}$ and $300 \mathrm{kHz}$. In the experiment, the temperature of the MIP structure reaches $110{ }^{\circ} \mathrm{C}$ while the MIO structure only reaches $80{ }^{\circ} \mathrm{C}$ at full load conditions after 30 minutes continues operating at a $23{ }^{\circ} \mathrm{C}$ room temperature (at resonant frequency of $180 \mathrm{kHz}$ ); the efficiency of the MIO structure and MIP structure are $90.3 \%$ and $93.7 \%$ respectively.

TABLE I

THE $350 \mathrm{~W}$ LLC TRANSFORMER AND CONVERTER SPECIFICATIONS

\begin{tabular}{cll}
\hline \hline Symbol & Parameters & Requirements \\
\hline$P_{\text {out }}$ & Power rating & $350 \mathrm{~W}$ \\
$V_{\text {in }}$ & Input voltage & $60 \mathrm{~V}(\mathrm{DC})$ \\
$V_{s}^{\prime}$ & Output voltage & $390-480 \mathrm{~V}(\mathrm{DC})$ \\
$V_{\text {nomial }}$ & Nominal voltage & $400 \mathrm{~V}(\mathrm{DC})$ \\
$f_{r}$ & Resonant frequency & $180 \mathrm{kHz}$ \\
$f_{s}$ & Switching frequency & $96 \sim 236 \mathrm{kHz}$ \\
$\mathrm{N}$ & Transformer turn ratio & $3: 20$ \\
$\mathrm{Q}$ & Quality factor & 0.36193 \\
$\mathrm{G}$ & Gain & $0.97 \sim 1.2$ \\
$L_{s}$ & Resonant inductor & $2.45 \mu \mathrm{H}$ \\
$L_{p}$ & Magnetizing inductor & $19.61 \mu \mathrm{H}$ \\
$C_{s}$ & Resonant capacitor & $319 \mathrm{nF}$ \\
\hline \hline
\end{tabular}

The transformer is designed to handle $72 \mathrm{~V}$ (RMS) square wave input at the most difficult operating frequency of 100 $\mathrm{kHz}$ with the maximum flux density $B_{\max }$ of $320 \mathrm{mT}$. The saturation of the transformer should not occur at resonant frequency based on previous experience. However, an input voltage impulse waveform was captured for the MIO prototype transformer, in that the peak voltage overshoot exceeds $100 \mathrm{~V}$. To investigate this saturation phenomenon, another prototype transformer with a separated leakage inductance was built for comparison. Under the same testing condition, this transformer has a maximum operating temperature of $75^{\circ} \mathrm{C}$ and the efficiency of $94.1 \%$.

Therefore, the transformer saturation of the MIO structure is now determined based on the above experiment due to the non-linear characteristic of the magnetic material. A regional saturation occurs in the insertion magnetics of the MIO prototype, in which it results in a further transformer saturation phenomenon (because the nonlinearity of the leakage inductance). When saturation is occurred, the core losses increase in an exponential manner with the increased flux densities. On the other hand, the transformer saturation also results in higher magnetizing current and increasing the copper losses dramatically.

For the illustration of the saturation phenomenon, the excitation current and load current of the circuitry need to be identified before going any further. In Fig. 2 (b), the equivalent load resistance can be obtained with the use of the Finite Harmonic Analysis Method [9] as $R_{e q}=8 / \pi^{\wedge} 2 R$ (refer to Fig. 2 (a) and (b) for definition). Thus, the load current $I_{e q}$ is now represented as the voltage across the transformer primary side over the $R_{e q}$, or with reference to the transformer turns ratio $N$ and the actual output current $I_{o}$ as:

$$
I_{e q}=\left[\frac{\pi I_{o}}{\sqrt{2 \cdot N}}\right]^{2}
$$

The magnetizing current can also be obtained since the voltage across the transformer primary side is known. By taking the forward voltage $V_{F}$ across the output rectifier and operating frequency $f$ into account, the result is:

$$
I_{p}=\left[\frac{N \cdot\left(V_{o}+2 \cdot V_{F}\right)}{4 \sqrt{2} f L_{p}}\right]^{2}
$$

Since the operation of LLC is frequency dependent, ( 4 ) is rewritten by taking account of the operating frequency and flux density of the magnetics based on Faraday's Law as:

$$
I_{p}=\frac{K_{a} \cdot B \cdot l}{\mu \cdot 2 \pi \cdot N}
$$

where for square wave input, $K_{a}=4$, sine wave input, $K_{a}=4.44$; $l$ is the mean path length of the magnetic core used and $\mu$ is the permeability. Summing ( 3 ) and ( 5 ), the maximum peak current $I_{\text {peak }}$ flowing through $L_{s}$ and $C_{s}$ is obtained as $I_{\text {peak }}=I_{e q}+I_{p}$. Applying (1) to ( 5 ) to the FEM program, the nonlinear phenomenon of the insertion type integrated magnetics is now ready for solving.

Due to the characteristics of series parallel type resonance operations, the $I_{p}$ is very large compared to other switching topologies in order to discharge the MOSFET capacitance within a short time (this guarantees the ZVS condition during the switches at on stage). As a result, it rises up the $I_{\text {peak }}$ and 
consequently results in higher conduction losses. The computation result with regards to the peak current versus $B_{\max }$ is shown in Fig. 3. The $B_{\max }$ of the MIO structure is $73 \%$ higher than that of the MIP structure under $90 \%$ load conditions, and it starts saturating when the load is further increased. The no-insertion structure has the least $B_{\max }$ due to the characteristics of the air-medium, but it does not have any ability to regulate the leakage inductance $(0.1 \mu \mathrm{H}$ is obtained). If the load is further increased to $700 \mathrm{~W}$, the MIP structure will eventually saturate with a sudden efficiency drop. On the other hand, this computation result also explains the nonlinearity of the MIO prototype while the MIP prototype has no issue for the $350 \mathrm{~W}$ LLC converters.

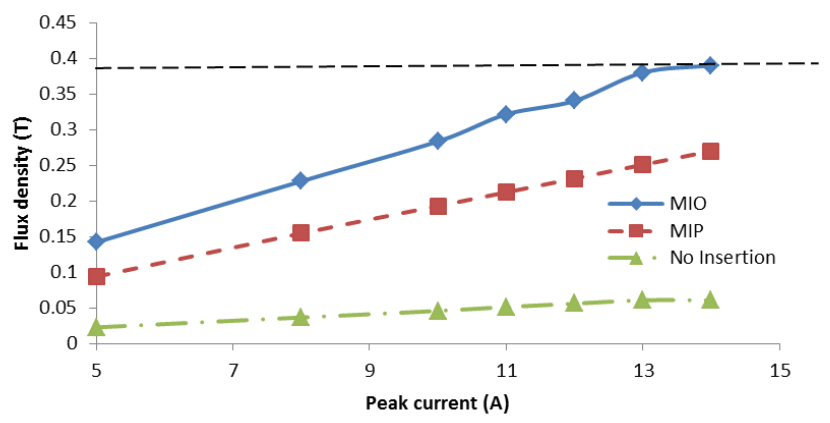

Fig. 3. Comparison of $B_{\max }$ under various load conditions

\section{THE REGIONAL SATURATION PHENOMENON}

\section{A. Nonlinear Phenomenon of the Main Core}

The nonlinear behaviour of the main cores is mainly attributed to the under-estimation of the number of winding turns. When designing a LLC transformer, it must be designed to withstand its most difficult condition. This is when the converter operates at the lowest switching frequency while having the greatest voltage gain. On the other hand, the inherent disadvantage of using the EE cores should be noticed, in that the magnetics get saturated easier at some specific regions (as marked in Fig. 4). The EE shaped magnetic cores tend to saturate at their corner edges as well as in the air-gap region. In particular, the middle leg of the transformer core performs more nonlinearly if the transformer is designed to be too close to its limitation compared to the other two legs. Cores in other shapes do not have this issue, but E cores suit today's power applications better due to their flat form.

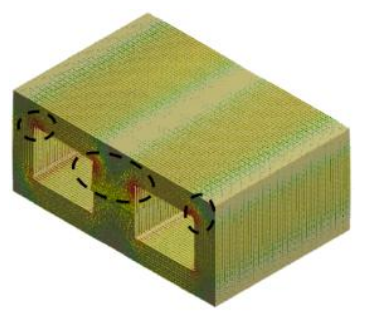

(a) Open circuit

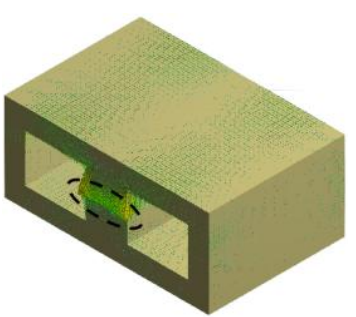

(b) Short circuit
Fig. 4. High flux density region of the MIO structure

\section{B. Nonlinear Phenomenon of the Magnetic Insertions}

The visualized flux density simulation result under full load conditions is shown in Fig. 5. The saturation of the MIO structure happens in a small region as shown in the result. The result also shows that the $B_{\max }$ of the MIO structure is $53.51 \%$ higher than the MIP structure. In the MIP structure, the leakage energy is stored more evenly in the air and its magnetic insertions. In comparison, the leakage energy tends to congest at the inter-connection area of the magnetic insertion and the main core for the MIO structure (at the spot where the flux density exceeding $0.4 \mathrm{~T}$ ).

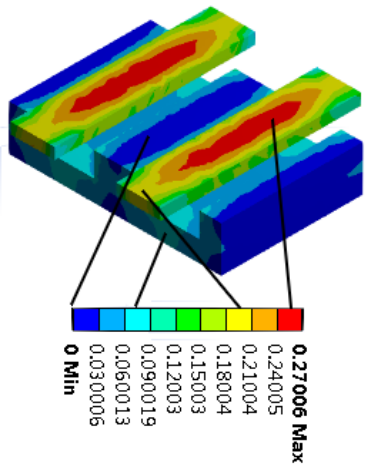

(a) MIP structure
Fig. 5. Flux distribution of insertion integrated magnetics under full load conditions

\section{ANALYSIS AND DISCUSSION}

The inherent leakage inductance of the transformer does not have an issue with magnetic saturation, nor air-core leakage inductance. The only problem with having a higher leakage inductance is that it reduces the coupling between the primary and secondary windings and brings down the transformer efficiency as a result. However, this inherent leakage inductance is not enough due to the requirements of the LLC topology. In practice, the design of the LLC converter requires a great leakage inductance in order to operate at a low frequency. For this reason, additional magnetic pieces are usually used to increase the leakage inductance, but this also creates the nonlinear phenomenon as shown in Fig. 5. Nevertheless, what is the consequence if the nonlinear phenomenon of magnetics has occurred? More specifically, how is it causing the destructive damage to the circuit? To investigate it, a SPICE simulation was conducted according to the circuit parameters tabulated in TABLE I.

The simulation result is shown Fig. 6 with the leakage inductors operating under at different saturation phenomenon. The non-saturation phenomenon represents the ideal functionality of the leakage inductance, in which it performs in a perfect linear behavior. For example, with the case study shown in this paper, the leakage inductance is maintained at $2.45 \mathrm{uH}$ at any condition. Under the 50\% saturation phenomenon, the leakage inductance reduces and thus the resonant frequency is moving upward with the increased load condition. Once the leakage inductor eventually saturates, only the inherent air-core leakage inductance takes effective. Thus, a very large surge voltage will be induced each time when input voltage changes its polarities. This surge in voltage can easily kill components within the implemented applications because the voltage rating is exceeded. This also results in a further saturation of magnetics such as the transformer and other magnetic components. 


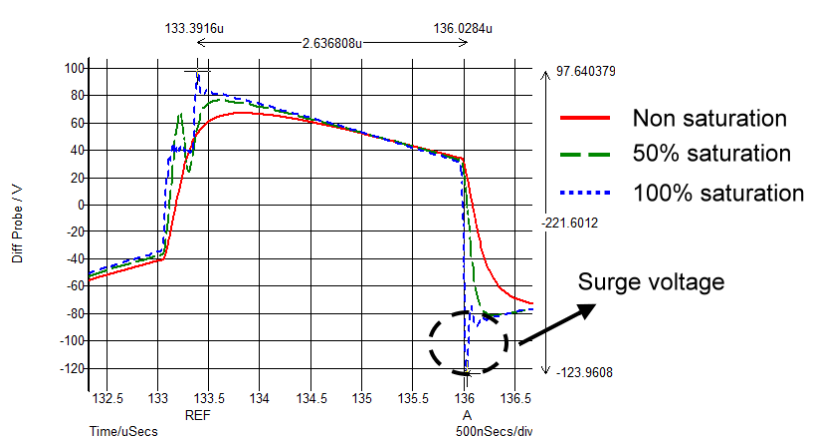

Fig. 6. The transformer input voltage waveform at $180 \mathrm{kHz}$ (at resonance)

The surge voltage reaches a peak voltage of $120 \mathrm{~V}$ at resonance, whereas it reaches a further level of $150 \mathrm{~V}$ at the most difficult conditions, as shown in Fig. 7. In order to achieve the maximum gain output, the converter needs to operate at around $100 \mathrm{kHz}$ at full load conditions. Thus, the surge voltage of $100 \%$ saturation phenomenon is now 1.43 times greater than the non-saturated phenomenon. Moreover, simulation results have shown here only focus on the analysis under full load conditions. Under light load conditions, the surge voltage is going to be even greater at above $1 \mathrm{kV}$.

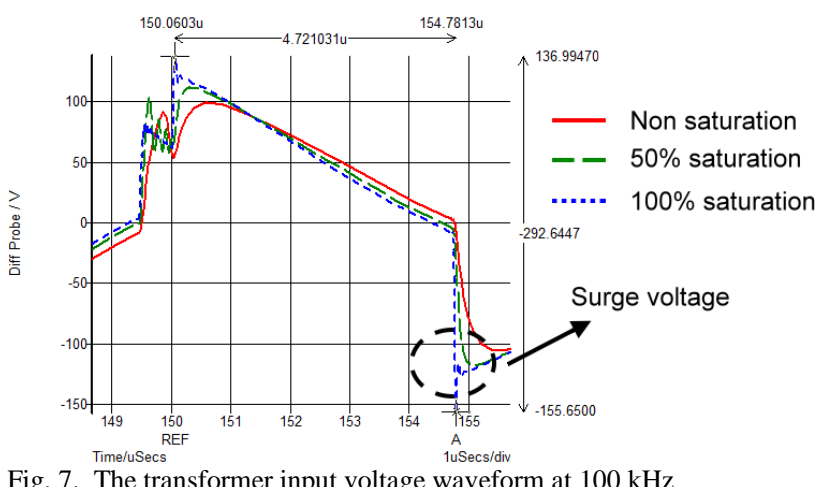

Fig. 7. The transformer input voltage waveform at $100 \mathrm{kHz}$

A further analysis was conducted to discuss the saturation with a focus on the magnetic insertion in the MIO structure. The saturation flux density of TP4A is about $0.4 \mathrm{~T}$ at $100{ }^{\circ} \mathrm{C}$, and thus the simulation parameters were aligned to this prerequisite. The output power is assumed at $1 \mathrm{~kW}$ in more extreme conditions to illustrate how magnetics are saturated with a more demonstrable visualized effect. The simulation result is shown in Fig. 8, in which the flux density has been marked in red if greater than $0.4 \mathrm{~T}$ (the air-gap distance between the insertion pieces and the main core is noted in the bracket below the inductance value). The magnetic with a leakage inductance of $2.45 \mathrm{uH}$ is completely saturated ( $>90 \%$ of areas in red). In comparison, the magnetic with the leakage inductance of $1.06 \mathrm{uH}$ is only saturated in a small region. The results show that the operation of the LLC should be bound to a higher minimum frequency when the power rating is high (assuming the dimension of the magnetics are not changed). Once the saturation of the inserted magnetic has occurred, other magnetic components also become saturated and consequently a surge in voltage is produced. Although the application does not burn out due to the surge in voltage, it will eventually overheat from the enormous magnetizing current.

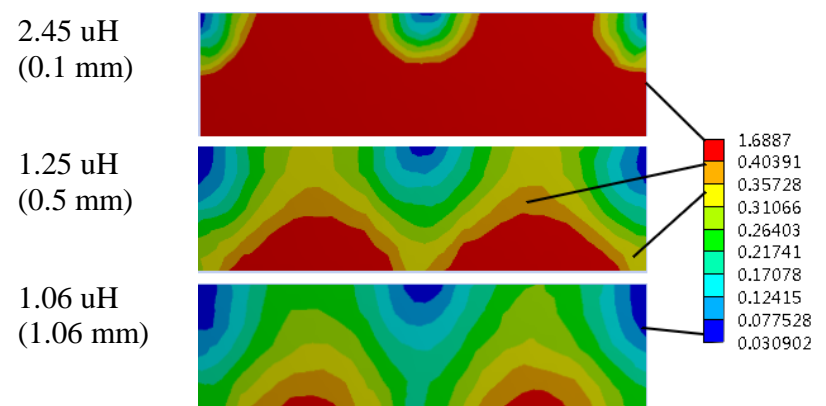

Fig. 8. Comparison of flux density among different leakage inductances of the MIO structure

To prevent the inserted magnetic integration from operating nonlinearly, several options can be implemented based on the design requirements. These options include: (1) increase the thickness of magnetic insertions; (2) increase the distance between the primary and secondary windings; (3) change the distance between the core and magnetic insertion (MIO structure); and (4) adopt the parameters of the LLC circuitry. In summary, this paper investigates and explains the nonlinear phenomenon of the inserted type integration magnetics used in LLC converters. The analysis and discussion were conducted using the FEM technique along with the mathematical calculations and SPICE simulation tools. For low input voltage and high power applications, magnetic designs for both the MIO structure and the MIP structure need to be carefully considered to avoid from the non-linearity behaviour.

\section{REFERENCES}

[1] D. Cheng, X. Dehong, Y. Zhang, C. Yi, Y. Okuma and K. Mino, "Impact of Dielectric Material on Passive Integration in LLC Resonant Converter," in PESC 2008. IEEE Power Electronics Specialists Conference, pp. 269-272, June. 2008.

[2] B. Lu, W. Liu, Y. Liang, F. C. Lee and J.D. Wyk, "Optimal Design Methodology for LLC Resonant Converter," in APEC. $21^{s t}$ Аnnu. Applied Power Electron. Conf., pp. 19-23, Mar. 2006.

[3] Z. Yanjun, X. Dehong, M. Kazuaki and S. Kiyoaki, "1MHz-1kW LLC Resonant Converter with Integrated Magnetics," in APEC. $22^{\text {nd }}$ Annu. Applied Power Electron. Conf., March. 2007, pp. 955-961.

[4] S. Stegen, and J. Lu, "Structure Comparison of High-Frequency Planar Power Integrated Magnetic Circuits, "IEEE Trans. on Magnetics, vol. 47, No. 10, pp. 4425-4428, Oct. 2011.

[5] E. Kim, Y. No, S. Lee, B. Chung, J. Lee, M. Park and D. Huh, "A Low Profile LLC Resonant Converter Using Novel Planar Transformer," in 27th Аппи IEEE. Applied Power Electron. Conf., Feb. 5-9, 2012, pp. 2232-2237.

[6] R. Chen, J. T. Stridom and J. D. van Wyk, "Design of Planar Integrated Passive Module for Zero-Voltage Switched Asymmetrical Half Bridge PWM Converter," in $36^{\text {th }}$ IAS Annu. Meeting. IEEE Industry Applications. Conf., Sept. 30-Oct. 4, 2001, vol. 4, pp. 2232-2237.

[7] Sihun. Yang, S. Abe and M. Shoyama, "Design Consideration of Flat Transformer in LLC Resonant Converter for Low Core Loss,"Power Electron. Conf., 2010, vol. 1, pp. 343-348.

[8] W. Water and J. Lu, "Improved High-Frequency Planar Transformer for Line Level Control (LLC) Resonant Converters," IEEE Magn. Lett, vol. 4, pp. 6500204, Nov. 2013.

[9] W. C. Ju, "LLC DC/DC Resonant Converter with PLL Control Scheme," M.S. thesis, Electr. Eng., National Cheng Kung. Univ., Tainan, Taiwan, 2006. 